\title{
Summary of April 2005 ANSI/NIST Fingerprint Standard Update Workshop
}

R. Michael McCabe

Michael D. Garris 
NISTIR 7242

\title{
Summary of April 2005 ANSI/NIST Fingerprint Standard Update Workshop
}

\author{
R. Michael McCabe \\ Michael D. Garris \\ Information Technology Laboratory \\ Information Access Division
}

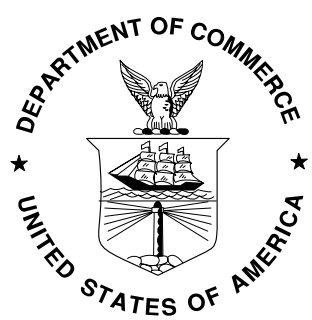

U.S. DEPARTMENT OF COMMERCE Carlos M. Gutierrez, Secretary TECHNOLOGY ADMINISTRATION

Michelle O'Neill, Acting Under Secretary of Commerce for Technology NATIONAL INSTITUTE OF STANDARDS AND TECHNOLOGY 


\title{
Summary of April 2005 ANSI/NIST Fingerprint Standard Update Workshop
}

\author{
R. Michael McCabe and Michael D. Garris \\ Image Group, Information Access Division, Information Technology Laboratory \\ National Institute of Standards and Technology, Gaithersburg, MD 20899
}

\begin{abstract}
This workshop ${ }^{1}$ was convened to chart the future of the ANSI/NIST-ITL 1-2000 fingerprint data exchange standard. This report is intended to provide a summary of that workshop. The current and future requirements and capabilities of existing stakeholders were first examined. Developments in information technology including extensible Markup Language (XML) were described and their potential relationship to the standard demonstrated. Approaches were presented for harmonizing this standard with other biometric standards. Additional proposals for improving the standard were introduced and a roadmap to upgrading the current version of the standard was generated.
\end{abstract}

Keywords: fingerprints, face, XML, FBI, standards, compression, JPEG, scan resolution

\section{Introduction}

The National Institute of Standards and Technology (NIST), in cooperation with the Federal Bureau of Investigation (FBI), conducted an ANSI/NIST Fingerprint Standard Update Workshop at NIST in Gaithersburg Maryland on April $26^{\text {th }}-28^{\text {th }}, 2005$. It was attended by over 120 representatives from federal, state, and local government agencies, Automated Fingerprint Identification System (AFIS) and related biometric technology vendors, consultants, and other interested parties. This workshop was held to review the existing American National Standards Institute (ANSI) standard for the electronic exchange of fingerprint and other identification information titled "Data Format for the Interchange of Fingerprint, Facial, \& Scar Mark \& Tattoo (SMT) Information" (ANSI/NIST-ITL 1-2000) ${ }^{2}$. The goal of the workshop was to:

- Determine if the current version of this standard should be withdrawn due to obsolescence or lack of use

- Reaffirm the standard in its present form, or

- Create a new version with enhancements and additional features.

The three day meeting can be viewed as four related parts. The first part reviewed existing systems and requirements, including overviews by the major stakeholders of

\footnotetext{
${ }^{1}$ http://fingerprint.nist.gov/standard/index.html

2 Available from http://www.itl.nist.gov/iad/vip/fing/fing.html ; also referenced as NIST Special Publication SP 500-245
} 
their operations and application of the ANSI/NIST standard. Emerging services by the FBI and a description of new processing initiatives from NIST were also presented. The second part reviewed the existing ANSI/NIST-ITL 1-2000 standard and its relationships to the work being accomplished in the ANSI INCITS $\mathrm{M}^{3}$ biometrics committee. The third part focused on advances in technology. A detailed panel discussion addressed a roadmap for the potential merging of $\mathrm{XML}$ into the next version of the standard. Additional contributions were introduced for the use of third level friction ridge details for automated matching of fingerprints and requirements for higher scanning resolution. The final part of the workshop was devoted to presenting specific proposals, evaluating each proposal, and determining the subsequent actions to be taken regarding their incorporation into the standard. Copies of each presentation can be viewed and/or downloaded from: http://fingerprint.nist.gov/standard/index.html .

\section{Existing Applications and Requirements}

Vance Hitch, the Chief Information Officer of the Department of Justice (DOJ), provided the opening remarks for the workshop. He set the stage for the workshop by stressing that the DOJ has a greater need for technology and standards than ever before. This requirement for data, fingerprint, and other biometric standards is the key to interoperability. Developed standards will provide the vehicle to coordinate efforts with federal agencies including Department of State (DOS), Department of Homeland Security (DHS), Department of Defense (DoD), and "international partners" such as the Royal Canadian Mounted Police (RCMP) and Interpol for prosecuting crime and protecting against the threat of terrorism.

\subsection{Major Stakeholders}

This opening was followed by a panel of major ANSI/NIST standard stakeholders. Each panelist highlighted the importance of the standard by providing overviews of their systems and describing the use of the data exchange standard in their normal operations.

- Gerd Hardt, the Interpol representative, stated that the ANSI/NIST standard is critical to the international exchange of fingerprint data. Since 1998 the Interpol AFIS Expert Group (IAEG) has promoted a standard method based on the ANSI/NIST standard for AFIS data exchange across country borders.

- Tom Hopper provided the FBI perspective stating that the standard facilitated transitioning to a paperless environment and it promoted the sharing of criminal history, fingerprint, and mugshot data at federal, state, and local levels. The Integrated Automatic Fingerprint Identification System (IAFIS) totally relies on the standard for the storage and processing of the 47 million criminal records online. These are searched against an average of $80 \mathrm{~K}$ transactions per day.

- Neal Latta from DHS and the US-VISIT program explained that the standard had been used for the transmission of 2.4 million criminal and 3.0 million civilian

\footnotetext{
${ }^{3}$ www.incits.org
} 
tenprint transactions to the FBI's IAFIS. This is in addition to the 24 million twofinger transactions processed against their own database since 2004.

- Charles Wilson(NIST) then presented an overview of the Patriot Act's mandate for biometric identification and verification testing that has been performed by NIST over the past two years. Many different levels of fingerprint image quality are included in the databases consisting of more than 128 million fingerprints from over 18 million subjects that are used in the testing programs. Conclusions from the FpVTE[1], SDK[2], and IDENT[3] tests were presented.

\subsection{New Processing Capabilities}

The FBI's IAFIS was put into full operation in July, 1999. It is now scheduled to be enhanced with a series of upgrades. FBI staff members overviewed these changes.

- Tracy Pacoe(FBI) reported on initiatives contained in the "Next Generation IAFIS" program. Major features included the automation of quality control checks in transaction submissions, an advanced interstate photo mugshot system, and a national palm print system.

- Scott Swann(FBI) went into more detail on the national palm print system which will have the functionality to receive, store, and search palm prints. The new system will also encompass "major case" prints. The point was brought up that the standard will have to be expanded to include codes, descriptions, and dimensions for these types of prints.

- Tom Hopper concluded the FBl's new initiatives with a description of a new service for civil background checks. This is based on the simultaneous capture using a tall sensor of the four sequence flats from each hand and the single capture of the two flat thumbs. Each finger is segmented and a quality score assigned to it. The Type-14 record will be used for the submission of the composite flat images. IAFIS will separate the four flats into individual images based on coordinate positions determined during the segmentation process. Advantages of this new process will include ease of use, better registration, and elimination of finger sequence errors.

\subsection{Requirement for Fewer Fingerprints}

Neal Latta (DHS) discussed the increasing popularity and advantages of systems relying on fewer than ten fingerprints. Two-finger systems are being used for applications including border control, mobile law enforcement, social services distribution, driver licenses, and voter registration. Advantages include low cost scanners, subject acceptance, less bandwidth, and lower system cost. However, disadvantages associated with this form of processing can be summarized as a lack of interoperability, a lack of scanner compliance standards, and a reduction in the amount of information available as it affects accuracy statistics. Latta suggested that new efforts should be started to overcome these problems. 


\subsection{HSPD \#12 Issues}

Charles Wilson gave a brief description of the Homeland Security Presidential Directive \#12 (HSPD \#12) ${ }^{4}$. This directive mandates the development of a common biometrically enabled Personnel Identification Verification (PIV) card. The card shall be used by all federal employees and contractors for physical and logical access to federal facilities and computers. FIPS 201 is a Federal Information Processing Standard for the PIV card and processes. NIST Special Publication 800-76 contains the required fingerprint and optional face technical biometric specifications for the FIPS 201 including the NIST Fingerprint Image Quality (NFIQ) value[4]. NIST Special Publication 800-73 contains the interface specifications for the FIPS 201. Existing FBI transactions will be used for enrollment and background checks while two fingerprints will be put on the PIV card itself for verification purposes.

Elham Tabassi (NIST) discussed the derivation and use of the first and only publicly available vendor-independent fingerprint image quality algorithm. The NIST Fingerprint Image Quality (NFIQ) assessment algorithm will be used for the PIV card. It is based on the premise that the performance of a fingerprint matcher is directly affected by the quality of fingerprint images captured and present in the database. The feature extraction portion of the algorithm computes the appropriate image fidelity characteristics. This results in an 11-dimensional feature vector that is input to a neural network. The neural network then classifies the feature vector into one of five levels of quality. An integer quality number between 1 (highest quality) and 5 (lowest quality) is assigned to each image. Fifteen fingerprint matching algorithms and 22 different datasets were used to evaluate the NFIQ algorithm and determine its reliability.

Walter Hamilton, representing the International Biometrics Industry Association (IBIA), provided the biometric industry's perspective on the PIV card. His main criticism of the PIV procedures was that use of fingerprint images for on-card storage is not appropriate. He was adamant that standards-based fingerprint minutiae templates should be stored on the PIV card. Hamilton's position was based on a limited amount of storage space on the PIV card, performance issues associated with data transfer of fingerprint images, timing issues, and privacy concerns. Acknowledging the current situation that template interoperability has not yet been established in widespread deployments, he suggested that the PIV development be delayed until further data confirms that template interoperability is achievable.

\section{Other Biometric Standards}

The next session of the workshop compared the ANSI/NIST-ITL 1-2000 standard to other developed biometric format standards. Fernando Podio (NIST) provided an overview of the national and international biometric standards activities in this area. He pointed out that the InterNational Committee for Information Technology Standards (INCITS) and NIST-ITL are both standards developer organizations certified by ANSI to develop standards in an open and fair manner. $M 1$ is the INCITS committee for biometrics that has developed commercial standards for the exchange of fingerprint, face, and iris information for identification and verification purposes.

\footnotetext{
${ }^{4}$ http://csrc.nist.gov/piv-project/index.html
} 


\subsection{ANSI/NIST-ITL Standard}

The ANSI/NIST-ITL standard was and is primarily intended for use by law enforcement agencies. It specifies the format for the exchange of descriptive text, fingerprint, face, scar, mark, and tattoo information to be used for the identification of a subject. An ANSI/NIST file can contain all of the available information pertaining to a subject including personal descriptive data, criminal history, and fingerprint and/or face data.

\subsection{CBEFF}

A primary difference between the M1 standards and the ANSI/NIST standard is that all of the M1 data format standards require that the defined biometric record be encased within a Common Biometric Exchange Formats Framework (CBEFF)[5] envelope. Cathy Tilton (Saflink) provided a brief description of CBEFF. While not constraining the encoding of data, it defines basic fields used by biometric data, and provides for the registration of biometric data via IBIA. The high level CBEFF structure consists of a header block, a biometric data block, and an optional signature block.

\subsection{Developed M1 Format Standards}

Overviews of the five developed M1 format standards were presented to determine points of contrast with the finger image, finger minutiae, and face standard counterpart records in the ANSI/NIST-ITL standard. One objective of the workshop was to attempt to harmonize the ANSI/NIST with the M1 set of standards.

Mike McCabe (NIST) addressed the M1 Finger Image-Based[6] and M1 Finger MinutiaeBased[7] standards both of which require a CBEFF wrapper. The M1 image standard contains a field that specifies various image acquisition parameters. The choice of this setting is commensurate with system and application requirements. The ANSI/NIST standard dictates minimum requirements that exceed those of the M1 image format. The M1 format is a compact binary form not readily expandable. The tagged field format used in the ANSI/NIST image records require more storage but is expandable.

Comparison of the M1 finger minutiae format with the ANSI/NIST format shows that one of the main differences between the two is that the M1 format provides specifics for the positioning of the minutiae. Another difference is that the M1 format uses a compact binary format to describe each minutia. This is in contrast to the ASCII format used by ANSI/NIST. There are also other minor differences between the two formats including the capability of the $\mathrm{M} 1$ format to accommodate encoded vendor proprietary data. At the end of the workshop McCabe also gave a brief description and status of the MINEX04 ${ }^{5}$ program. This evaluation test seeks to determine the current state of interoperability between AFIS vendors when minutiae templates are exchanged. The templates are based on the M1 minutiae format. A report on this project is targeted for February 2006.

Cathy Tilton described the M1 Finger Pattern-Based[8] standard. Its use is intended for portable devices such as the slide format sensors. Rather than encode minutiae, the pattern approach analyzes global sections of the image. A data and resolution reduction technique is first applied. The resultant image is then broken into cells, each of which can be represented by a sinusoidal waveform.

\footnotetext{
${ }^{5}$ http://fingerprint.nist.gov/minex04/index.html
} 
Jim Cambier (Iridian) discussed the Iris[9] interchange format that has been developed in M1. It is used for the exchange of iris information among multiple vendors and applications. There are two image storage formats that are specified by this M1 standard - the rectilinear and polar formats. The rectilinear format is essentially a raw image format with a minimum of preprocessing. A cropped compressed iris image would require $12 \mathrm{~K}$ to $15 \mathrm{~K}$ bytes of storage. The polar format preprocesses the iris image to find the pupil and iris boundaries. The iris portion of the image is then converted to polar coordinates and typically stored in $2 \mathrm{~K}$ bytes of storage.

The final M1 format was presented by Paul Griffin (Identix). Although there is a face record type in the ANSI/NIST format, the M1 Face Recognition[10] format contains more detail. The M1 facial standard is image-based. A face template does not exist at this time as there has not been significant agreement on the contents of a possible facial template. The standard specifies best practices for how the face should appear rather than the mechanics of capturing the photograph. In order to improve recognition performance the standard allows for the inclusion of additional visible information pertaining to the face that can be discernable to an operator (for example eye color). Optional feature points (such as the specification of eye positions) are also part of the M1 standard. Both JPEG[11] and JPEG 2000[12] algorithms can be used for compressing facial images. However, too much compression can result in compression artifacts and a decrease in search accuracy.

\subsection{M1-Style ANSI/NIST Record}

Currently, there is no counterpart for the fingerprint pattern, iris, and other unique M1 biometric data formats within the ANSI/NIST standard. However, an ability to incorporate such M1 data formats within the standard framework would be useful. This can be accomplished with the creation of a different ANSI/NIST record type for each new biometric that is defined. Alternatively, an approach can be developed that will allow the M1 formats to be included within the ANSI/NIST framework. As both of these are viable approaches, there is nothing to prohibit the inclusion of both.

Dale Hapeman, representing the DoD Biometrics Management Office (DoD/BMO), stated that there is a need for multiple biometric data types in the ANSI/NIST standard to support existing and deployed DoD systems in the field. This could be accomplished by the creation of an additional record that would support all the M1 data formats contained within a CBEFF structure.

This recommendation for the creation of an M1/CBEFF record initiated considerable discussion. Such an approach would allow the ANSI/NIST standard to carry iris, voice, earlobe and other data without the need for the creation of a new record type for each biometric. However, the ANSI/NIST standard has the advantage of simplicity and a consistent parsing structure. This recommendation would introduce a complex system of possibilities that could cause a situation that the receiver of a transaction would not be able to parse an entire file transaction. At this time, most of DoD's requirements could be solved with the addition of a new iris record. It was decided to delay any action on the recommendation until the end of the meeting. 


\section{Advances in Technology}

\subsection{Use of XML with the ANSI/NIST Standard}

A four-member panel discussion on the use of XML (eXtensible Markup Language) with the ANSI/NIST standard presented several ways to merge XML principles with the standard. Gerry Coleman of the Wisconsin Department of Justice (WI/DOJ) was the panel moderator and presented a basic introduction to XML concepts. He pointed out that it was a generic text format for describing and tagging data. Its design goals included the creation of human-legible and reasonably clear documents - terseness in $\mathrm{XML}$ being of minimal importance. The purpose of XML was not to perform active functions (such as HTML's bolding function) but to structure, store, and send information. Like HTML, XML employs paired symmetrical tag names that are defined by the implementation and agreement between the sender and recipient. These tags can also be nested to create a "well-formed" XML document. As applied to the ANSI/NIST standard, Coleman proposed that field tag names could replace the current field numbers. For example, the Type-2 record length field "2.001:" could be converted into "<length $>$ " and "<llength $>$ " tags. Similarly, current character separators could be replaced with tag names and angle brackets.

There are good reasons to enhance the ANSI/NIST standard with the use of XML including readability, and interoperability. The disadvantage is that it is a text formatting convention that doesn't mix well with binary (image) data. Several proposals were mentioned for the handling of binary data. Image data could be embedded in the file by encoding it into a Base-64 block of data. But this results in a $33 \%$ increase in size and additional overhead in the encoding and decoding processes. A second approach was to provide a URL reference to an external file containing the image data. A third approach was the Message Transmission Optimization Mechanism (MTOM) whereby documents and attachments are packaged together for transmission.

Coleman serves as chair on the Joint Task Force on Rapsheet Standardization (JTF). The JTF has created an XML rapsheet transmission standard using tagged data similar to the ANSI/NIST fingerprint transmission standard and converted it to conform to the Global Justice XML Data Model (GJXDM). Following up on this work the JTF recommends that the FBI develop a Type-2 record specification using GJXDMconformant XML and that an XML version of the entire ANSI/NIST fingerprint standard be developed.

Catherine Plummer (SEARCH) began her presentation by stating that goals of Information Exchange Package Documentation (IEPD) include the support for interoperability among justice systems, expand and refine GJXDM/DD through experienced feedback to resolve vague definitions, and to limit the number of key choices to support interoperability. The steps involved with the IEPD process were described including domain modeling, GJXDM mapping, subset schema, sample XML instances, and packaging. She concluded her presentation by stating that the goal of IEPD is to exchange messages not to build databases.

Customers of Scott Hills (AWARE) have frequently requested conversion from ANSI/NIST to XML and to a much lesser degree conversion in the reverse order. In light of these requests, his recommendation is that the current standard should not be converted to a pure XML format. Guidelines or templates for converting between 
ANSI/NIST and XML should be developed for the revision of the ANSI/NIST standard. Two approaches were suggested. First, develop a domain-independent specification that would mirror the structure of the current standard and ensure that one schema would be appropriate for any ANSI/NIST file. Second, develop a domain specific schema that would reflect domain specific requirements for Type of Transactions (TOT), record types, and all Type-2 record requirements in the element tags. Although both approaches have been implemented, the notion of a "standardized translation" between the two is being promoted by Hills.

The last panel presentation was delivered by Alessandro Triglia (OSS Nokalva) who proposed an XML-based version of the ANSI/NIST standard. He began with an example of an ANSI/NIST formatted record converted to XML with CBEFF tags embedded. This was followed by a thorough review of the CBEFF status, a description of CBEFF concepts including a detailed description of the patron formats and the CBEFF structure. He concluded with a description of the features of an XML patron format, an example of it, and a brief description of a proposed document format.

A discussion period that followed the panel presentations was begun with Coleman stating that despite the several proposals offered, there did not appear to be a unified solution put forth by the panel.

Moving the ANSI/NIST standard toward XML would simplify the interaction between AFIS and non-AFIS (criminal history) systems. But a concern was raised about transition costs in terms of money and time to covert thousands of existing applications to XML. The added capabilities will drive the use of XML. It was suggested that the vendors won't drive users to XML. The users and the public will drive applications to Webservices, which will drive the migration toward XML. It is the users who will write $\mathrm{XML}$ requirements into their requests for proposals.

It was pointed out that existing equipment becomes obsolete and must be replaced but that legacy systems and data would continue to be supported. To resolve the cost concern is was proposed that both XML schema and ANSI/NIST files should be allowed to coexist. A suggestion was made to develop a second standard for XML that would "mirror" ANSI/NIST data.

Before the discussion ended it was repeated that the binary problem had still not been resolved. Dealing with the $33 \%$ increase in size and the latency overhead for encoding and decoding is a struggle. But it was stated that if you email ANSI/NIST records today, you are already using Base-64 encoding.

The participants at the workshop decided to delay further discussion and decisions specific to the roadmap to XML until the end of the workshop.

\subsection{Third Level Details}

Steve Meagher (FBI) opened the next session with two proposals submitted by the Scientific Working Group on Friction Ridge Analysis, Study and Technology (SWGFAST). The first was for the inclusion in the standard of level three details from fingerprint images and the second was a proposal for a minimum scanning resolution of 1000 pixels per inch (ppi) or 39.37 pixels per milllimeter (ppmm). 
Historically, development of AFIS technology was a result of a need to achieve a required throughput and performance for the high volume processing of tenprint fingerprints. This was achieved through the use of both classification matching and minutiae matching techniques. These are commonly referred to as level 1 details which include orientation, cores, and deltas, and level 2 details which includes combinations, relationships, and the absence of characteristics.

Although this approach has worked well for tenprint processing, the quality and amount of captured information may not meet all of the needs for latent print examination. Level three details are also needed by examiners to improve latent print matching. Third level details include ridge features such as dots, pores, ridge edge shapes, ridge widths, path information, and ridge relationships.

This information contributes to fingerprint image quality which is a key factor for improved latent matching. SWGFAST proposes that level three details be included as part of the Type-9 minutiae record. A survey was taken of the workshop participants regarding the addition of level three details. There was no objection to inserting this information into the standard. Meagher agreed to head up an ad hoc group to include SWGFAST and other interested parties to develop the required concepts, definitions, and representations of level three details.

The second part of their proposal states that in order for level three details to be useful it is necessary that the images be scanned at $1000 \mathrm{ppi}$ in order to detect the level three details. He also proposed that if the fingerprint images are to be compressed, then JPEG 2000 shall be used with a maximum compression ratio of 10:1. Like the other major controversial issues, the decision on this proposal was postponed until the end of the workshop.

\subsection{Facial Image Compression}

Paul Griffiin (Identix) presented a talk on the trade-offs between accuracy and compression for face recognition. When the compression ratio increases, the facial recognition accuracy degrades. In a study that he summarized, recognition accuracy dropped by $20 \%$ as the file size dropped from 150KB bytes to $7.5 \mathrm{~KB}$.

However, there are applications that require a captured facial image to be overcompressed in order to store it on particular media such as a smart card. To compensate for this trade-off, a region of interest (ROI) compression approach can be used to preserve recognition performance while meeting file size requirements. The image can be compressed as two or three nested areas. For the case of two ROls, the inner area encompasses the hair, chin, and neck of a subject. The outer area consists of the remainder of the image. A third ROI or core area can be defined to include just the forehead, eyes and nose. If two ROls are used, the outer area is compressed more aggressively than the inner area. For three ROls, the core area would be the least compressed, with the surrounding ROI (the inner area) more compressed, and the outer area the most aggressively compressed. By using this approach nearly a 3:1 reduction in file size can be achieved without any loss in performance accuracy.

\subsection{Fingerprint Image Compression}

Tom Hopper (FBI) then addressed the compression of fingerprint images scanned at 1000 ppi. The FBI developed the Wavelet Scalar Quantization (WSQ) algorithm for 
compressing fingerprint images scanned at 500 ppi (19.69 ppmm). The use of wavelets was found to be the most efficient technique for this application. However, when the same WSQ algorithm was applied to images scanned at 1000 ppi, the algorithm did not function as expected. Rather than devoting additional resources to upgrade the WSQ algorithm, the JPEG 2000 algorithm was chosen as an alternative. Already a standard, it is similar to the WSQ, wavelet based, efficient, and produces excellent image quality. A JPEG 2000 profile for 1000 ppi fingerprint images has been developed by Margaret Lepley (MITRE)[13]. This profile is a restricted subset of JPEG 2000 parameter settings to insure image quality and interoperability. It also provides a path for transcoding 1000 ppi JPEG 2000 images to 500 ppi WSQ images. Use of JPEG 2000 with the 1000 ppi profile will be included in the FBI's Electronic Fingerprint Transmission Specification (EFTS). Certification criteria for proper adherence to this approach will also be developed in the near future.

\section{Proposed Updates and Action Items}

The remainder of the workshop addressed specific changes and enhancements proposed for inclusion in the ANSI/NIST-ITL 1-2000 standard. Mike McCabe set the stage for discussing proposed updates to the standard by presenting a summary of the uses for the standard, its history, its basic record structure, and the use of fields within the records.

Following this introduction, specific recommendations for updating the standard were presented and reviewed. This included those issues which had earlier been postponed for further discussion and review. An appropriate amount time was allowed for discussion of each topic. In order to get a "sense" of the room regarding each item, workshop participants were asked for their opinion on the value of each proposal: should it be considered for inclusion in the standard? There was no formal voting protocol established for the workshop as the official procedures used to update the standard were not yet in effect. Therefore, the answers to these questions would not be binding. Rather, they would be used as a "starting point" or guide for the development of a revision to the standard. An informal process was used to determine the action to be taken on each proposal. It was usually accomplished by asking for objections to the assumption of inclusion in the standard. On more contentious issues, participants (one per organization) were asked to raise their hands to vote on an issue. Hands were counted but participants were not individually polled.

The reason for this informality was that during the course of the workshop it became very apparent that, by the end of the three days, full agreement on all open issues that had been introduced would not be achieved. The workshop did identify a list of topics and issues for possible inclusion in the draft for an updated standard. It would be necessary for most of these issues and new proposals to be better developed and refined in small ad hoc groups - each concerned with a specific topical area. Email and teleconferences would be the tools to accomplish their work outside of the workshop. Conclusions reached by each of these ad hoc groups shall be documented in a form acceptable for inclusion in a proposed draft revision of the standard. All proposals will be reviewed at the next workshop to be held at a later date. 


\subsection{Facial Image Proposals}

Paul Griffin presented a comparison between concepts and facial information included in the ANSI/NIST Type-10 record and those used by the ANSI INCITS 385-2004 Face Recognition Format. The inclusion of features and other information from INCITS 385 in the Type 10 record would provide additional capabilities for facial recognition and other applications. Several recommendations were made to enhance the Type-10 record.

- Allow the use of color JPEG 2000 as an image format to provide better image quality than traditional JPEG at a fixed compression.

- Add source type to aid in artifact removal and gamma correction.

- Add provision for quality score and algorithm identification information.

- Expand format to include facial feature points that function as the minutiae of the face.

- Define fields for the 3D pose angle set of yaw, pitch, and roll.

- Include a facial image capture application profile that covers compression limits, capture requirements, and other best practice attributes or requirements.

- Define additional fields for temporary face image attributes such as facial expression, and eye blinking.

There was a basic consensus from the workshop that these issues should be refined by a "FACE" ad hoc group and this group's recommendations should be presented at the next workshop.

\subsection{Character Sets, Codes, and Tracking}

Rob Mungovan (AWARE) proposed five recommendations to the ANSI/NIST standard. All were accepted to be sent to a "UTF/GPS" ad hoc group for refinement and inclusion in the draft standard update.

- Use UTF-8 in place of 7-bit ASCIl for all user-defined fields to simplify international applications. This recommendation elicited considerable discussion including the need for a registry of character sets.

- Expand the Type-10 record to include color JPEG 2000 and JPEG 2000 lossless modes as supported compression algorithms.

- Define a new field in the Type-1 record to identify the software used to generate the transaction. This field would include the vendors name, software application name, and version number of the software.

- Formally specify within the standard the name of each algorithm that can be used for compressing images in Type 13-16 records.

- Formally specify within the standard the value for WSQ and JPEG that can be used in the Type-4 record.

Bonnie Scheier (SABER) proposed two recommendations. Both proposals triggered discussion and unresolved questions. However, they were accepted in concept and sent to the UTF/GPS ad hoc group for further development.

- Develop a Global Positioning System (GPS) data field to be added to the Type-1 record so as to provide a mapping of an arrestee's location at or near the point of arrest. 
- Develop a Submission Tracking Field (STF) for the Type-1 record to support the tracking of submission's header information and timestamps while traversing multiple vendors and jurisdictions.

\subsection{Harmonizing Standards and Miscellaneous Updates}

Mike McCabe presented several recommendations aimed at harmonizing standards developed by ANSI/NIST with those developed by M1. Additional miscellaneous proposals to update existing tables and requirements were proposed. All of the recommendations were accepted and assigned to the "MISC" or the "SWGFAST/FBI" ad hoc group for additional refinement.

- Define and reserve a new block of fields for the Type-9 minutiae record to contain information similar to that found in the INCITS 378-2004 Finger Minutiae format. Minutiae position will be as defined according to the method used by the M1 format. This block of reserved fields shall be allocated for the same purpose as those blocks currently assigned to specific AFIS vendors.

- Define additional fields to the Type 13-16 records that will specify image capture parameters, information on the number of views, image quality information, and optional product identification.

- Define a new record type to contain iris image information. Jim Cambier (Iridian) will be asked to confirm that information fields contained in this record do not infringe on any existing patents.

- Add a code "15" to Table 6 in order to represent the simultaneous capture of two plain thumbs.

- Adjust the length and width dimensions in Table 6 (finger codes 11-15) to accommodate the enlarged platen sizes for plain images on the newer live scan devices.

- Add codes and dimensions for right and left thenar, hyperthenar, and interdigital areas of the palm. This proposal was expanded to include the codes, dimensions, and descriptions for each of the major case prints requested by SWGFAST.

- Add a vendor identification field for all image and minutiae records.

- Update table 5 describing impression types to include the swipe, contactless, and other impression types suggested by the SWGFAST/FBI ad hoc group.

- Increase the maximum size of the source agency/ORI to 50 characters for record types requiring this information.

\subsection{Level Three Details and Scanning Resolution}

Steve Meagher submitted the level three details and the resolution proposals on behalf of SWGFAST.

- The first recommendation called for establishing an ad hoc group to develop an approach to encoding third level details for fingerprints. These include, but are not limited to, definitions and descriptions of details including pores, ridge edge shapes, ridge widths, dots, and ridge relationships. The recommendation was approved. The "SWGFAST/FBI" ad hoc group shall consist of AFIS vendor representatives, SWGFAST members, and other interested participating volunteers. 
- The second proposal stated that the normal mode of finger and palm print image capture should be 1000 ppi (39.37 ppmm) minimum scan resolution. Images should be compressed using JPEG 2000, not to exceed 10:1 compression. Legacy compatible 500ppi (19.69 ppmm) image capture and processing should be permitted.

A "straw" vote was taken and the motion failed. The Type-14 record is being popularized as a civilian identification record for processing in FBI and DHS background checks. A requirement for a 1000 ppi scan resolution may cause non-law enforcement agencies to abandon use of the ANSI/NIST standard if it tied to latent forensic identification constraints. This proposal was then split into three separate parts being applicable to latent, tenprint, and palm print images. A second vote was taken that determined the minimum scanning resolution for the capture of latent images should be set to $1000 \mathrm{ppi}$. Two additional votes were taken to determine the scanning resolution for tenprint and palm print images. The result of these two votes was to maintain the status quo of $500 \mathrm{ppi}$ as a minimum but with a strong recommendation for 1000 ppi for tenprint and palm print images. If the fingerprint images scanned at $1000 \mathrm{ppi}$ are to be compressed, then JPEG 2000 shall be used with a maximum compression ratio of $10: 1$.

\subsection{M1 Data Formats}

Dale Hapeman revisited the additional CBEFF record structure and presented two alternatives for consideration.

- First, any CBEFF record format would be allowed to be included. It would not be patron-format specific. The CBEFF patron format owner and type information would be located at the beginning of the record. The remainder of the record would contain the CBEFF BIR (binary data).

- The second alternative would allow the ANSI/NIST to become its own patron format. To accomplish this approximately 23 CBEFF header bytes would be added at the beginning of the record. The biometric data would follow.

The second approach appeared to have the most support. It was also suggested that in order to provide broader application for the ANSI/NIST standard, a minimalist patron format could be created and put in all ANSI/NIST higher resolution records. The addition of a new record type to accommodate data structures defined in $\mathrm{M} 1$ but not in ANSI/NIST was accepted in concept. The issue was assigned to the "M1/CBEFF" ad hoc group for development.

\subsection{XML Issues}

Gerry Coleman introduced recommendations for an ANSI/NIST roadmap to XML. Two options were presented.

- Allow XML as "User-defined" data in the Type 2 descriptive text record. This implies retaining the existing structure and formatting mechanisms, but allowing $\mathrm{XML}$ instead of numeric tags within a single logical record. This may result in the creation of a new Type $2 \mathrm{~N}$ XML user-defined data record. 
- Define entire ANSI/NIST package by creating an XML structure for the entire specification. Tag names would be created for the entire package, each logical record, and all numeric tags. This would eliminate use of $f s, g s, r s, u s$ separators. Base64 encoding would be used for embedded binary data.

Some discussion followed. Workshop participants agreed that an "XML" ad hoc group should study the issues involved, and develop a solution for the draft of the updated standard.

\subsection{Live Scan Issues}

The final recommendations for updating the standard were submitted by Bill Long (Business Performance Research Associates). He provided an overview and comparison of next generation livescan devices and associated factors that may need to be considered for inclusion in the update of the standard. The following issues will be considered by the "LiveScan" ad hoc group.

- Modify table 6 of the standard to include 2-finger captures of plain fingerprint impressions. Although not explicitly stated in the standard, this capability is available in the Type-14 record by listing the two finger positions separated by the ASCII us or rs separator characters.

- Create an additional field to indicate the quality scale and quality value of the fingerprint image in Type-14 and above records.

- Create an additional field to indicate if a finger were "live" or not. As there are several issues associated with this topic it was decided to add a field to indicate whether the capture was in an "attended" or "unattended" mode.

- Addition of a field to represent 3-D livescan capture of fingerprints. The LiveScan ad hoc group will provide a concrete proposal on this subject for the next workshop.

\subsection{Security}

Greg Cannon (CrossMatch) raised the question as to whether the ANSI/NIST standard should be updated with applicable security and privacy measures. No decision was reached on the issue. But it was decided that a "SECURITY" ad hoc group should be created to look at the issues and provide a proposal or make a report at the next workshop.

\section{ANSI Standard Development Process}

The participants at the workshop favored updating the ANSI/NIST-ITL 1-2000 standard to incorporate new features that had been discussed. To accomplish this, procedures established by the American National Standard Institute (ANSI) would be used. Mike Hogan (NIST) provided information on ANSI and the ANSI requirements that NIST must follow for updating the standard. ANSI accredits its 200 standards developers based on approval of the procedures used by each developer. NIST/ITL has been accredited by ANSI since October 5, 1984, to develop standards and guidelines for information exchange relating to automatic data processing and related systems. 
One of the hallmarks of the ANSI process is that consensus must be achieved on a proposed standard by a group or "consensus body" that includes representatives from materially affected and interested parties. NIST/ITL uses the canvass method to establish consensus (i.e., the consensus body is the list of canvass members). NIST/ITL will issue a call for members to start development of the canvass list. Individuals and organizations that agree to be canvassees have an obligation to vote on at least the final version of the draft standard. There are established rules that govern the approval of a draft standard. A fairly detailed flowchart of the ANSI standards development process is available in the power point presentation for this topic.

\section{Conclusions}

This workshop was convened to determine the future for the ANSI/NIST-ITL 1-2000 standard. Should it be withdrawn for lack of use or relevance, reaffirmed for another five years, or updated and modified? Participants at the workshop decided it should be updated so as to fulfill additional application requirements, and to take advantage of advances in technology that have occurred since the 2000 version was approved. Workshop participants then had to decide which aspects of the standard were to be affected.

The first step in this process was to examine current and future requirements of existing stakeholders. The FBI, DHS, INTERPOL, and NIST described their current applications of the standard and how planned initiatives would require alterations to the existing standard. New developments in approaches to information technology such as XML, and new standards such as those developed by the INCITS M1 group were described. Their relationships to the ANSI/NIST standard were outlined and discussed. Possible methods of incorporating XML with the current standard were reviewed, as were approaches to harmonizing the ANSI/NIST standard with the M1 suite of standards. Finally, suggestions and enhancements aimed at specific aspects of the standard were proposed, discussed, and evaluated. Agreement for the inclusion of several proposed issues was achieved during the workshop. However, the majority of proposed issues required additional refinement or study to convert the ideas into an acceptable form for further review and acceptance. To accomplish this, several ad hoc groups were formed. Each was tasked with developing solutions to specific issues. One person from each ad hoc group was appointed as the chair. These chairs will be responsible for refining proposals and providing completed solutions for each issue.

The following is a list of the ad hoc groups appointed with a brief reference to the tasks of each group. The first name in each group has volunteered to serve as chair with the remaining members having expressed an interest in serving on the group. Membership in any of the ad hoc groups can be increased or changed by contacting the chair of the group.

- Face - Compression issues, 3D parameters, best practice

o Paul Griffin - Paul.Griffin@identix.com

o Patrick Grother

o Mike McCabe 
- UTF/GPS - UTF coding, GPS, Tracking, Vendor Identification

o Rob Mungovan - rob@aware.com

o Bonny Scheier

o Ralph Lessmann

o Tony Mislin

o Dale Hapeman

- MISC - Iris \& minutiae record enhancements etc.

o Mike McCabe - mccabe@nist.gov

o Rob Mungovan

o Dale Hapeman

o Paul Griffin

o Tom Hopper

o Bill Long

- SWGFAST/FBI $\quad-3^{\text {rd }}$ Level Details, Major Case Prints, Impression Types

o Steve Meagher - stephen.meagher@ic.fbi.gov

o Ben Bavarian

o John Burt

o Austin Hicklin

o Scott Swann

- Geppy Parziale

o Bill Long

- M1/CBEFF - Develop a record type to handle M1 data formats
- Dale Hapeman
- dale.hapeman@dodbfc.army.mil
o Bonny Scheier
o Rob Mungovan

- $\quad \mathrm{XML}$ - Develop a scheme that is compatible with standard
o Gerry Coleman
- coleman@DOJ.STATE.WI.US
o Ralph Lessmann
o Dave Weston
- David Woo
- Allessandro Triglia
o Rob Mungovan
- David Rodman
o Bonny Scheier 
- LiveScan - Encoding of 3D Fingerprint data

o Bill Long -

o Steve Meagher

- Security - Standard guidance issues

- Greg Cannon - greg.cannon@crossmatch.com

o Kevin Wilson

\section{References}

[1] Fingerprint Vendor Technology Evaluation (FpVTE) 2003 http://www.itl.nist.gov/iad/894.03/pact/pact.html

[2] Studies of One-to-One Fingerprint Matching with Vendor SDK Matchers, NISTIR 7221 http://www.itl.nist.gov/iad/894.03/pact/pact.html

[3] Matching Performance for the US-VISIT IDENT System Using Flat Fingerprints, May 2004. NISTIR 7110 http://www.itl.nist.gov/iad/894.03/pact/pact.html

[4] NIST Fingerprint Image Quality NISTIR 7151; http://www.itl.nist.gov/iad/894.03/pact/pact.html

[5] NISTIR 6529-A-2004, Common Biometric Exchange Formats Framework (CBEFF)

[6] Finger Image-Based Data Interchange Format, ANSI INCITS 381-2004

[7] Finger Minutiae Format for Data Interchange, ANSI INCITS 378-2004

[8] Finger Pattern Data Interchange Format, ANSI INCITS 377-2004

[9] Iris Image Interchange Format, ANSI INCITS 379-2004

[10] Face Recognition Format for Data Interchange, ANSI INCITS 385-2004

[11] ISO/IEC 10918, Information Technology - Digital Compression and Coding of Continuous-tone Still Images (JPEG) - Parts 1-4

[12] ISO/IEC 15444, Information Technology - JPEG 2000 Image Coding System Parts 1-10

[13] Profile for 1000ppi Fingerprint Compression, Version 1.1, April 2004, http://www.mitre.org/work/tech_papers/tech_papers_04/lepley_fingerprint/lepley_ fingerprint.pdf 